\title{
BMJ
}

\section{Association of suicide attempts with acne and treatment with isotretinoin: retrospective Swedish cohort study}

\author{
Anders Sundström, pharmacoepidemiologist, ${ }^{1,2,3}$ Lars Alfredsson, professor in epidemiology, ${ }^{4}$ Gunilla Sjölin- \\ Forsberg, dermatologist and clinical pharmacologist, ${ }^{2}$ Barbro Gerdén, dermatologist, senior expert, ${ }^{2}$ Ulf \\ Bergman, clinical pharmacologist, ${ }^{3}$ visiting professor, ${ }^{1}$ Jussi Jokinen, senior psychiatrist ${ }^{5}$
}

\begin{abstract}
${ }^{1}$ Centre for Pharmacoepidemiology Karolinska Institute, Karolinska University Hospital T2, 17176 Stockholm, Sweden

${ }^{2}$ Medical Products Agency, 75103 Uppsala, Sweden

${ }^{3}$ Division of Clinical Pharmacology, Karolinska Institute

${ }^{4}$ Institute of Environmental Medicine, Karolinska Institute

${ }^{5}$ Department of Clinical

Neuroscience, Psychiatry Section Karolinska Institute

Correspondence to: A Sundström Anders.Sundstrom@Ki.se
\end{abstract}

Cite this as: $B M J$ 2010;341:C5812 doi:10.1136/bmj.c5812

\section{ABSTRACT}

Objective To assess the risk of attempted suicide before, during, and after treatment with isotretinoin for severe acne. Design Retrospective cohort study linking a named patient register of isotretinoin users (1980-9) to hospital discharge and cause of death registers (1980-2001).

Setting Sweden, 1980-2001.

Population 5756 patients aged 15 to 49 years prescribed isotretinoin for severe acne observed for 17197 person years before, 2905 person years during, and 87120 person years after treatment.

Main outcome measures Standardised incidence ratio (observed number divided by expected number of suicide attempts standardised by sex, age, and calendar year), calculated up to three years before, during, and up to 15 years after end of treatment.

Results 128 patients were admitted to hospital for attempted suicide. During the year before treatment, the standardised incidence ratio for attempted suicide was raised: 1.57 (95\% confidence interval 0.86 to 2.63 ) for all (including repeat) attempts and 1.36 (0.65 to 2.50) counting only first attempts. The standardised incidence ratio during and up to six months after treatment was 1.78 (1.04 to 2.85) for all attempts and 1.93 (1.08 to 3.18) for first attempts. Three years after treatment stopped, the observed number of attempts was close to the expected number and remained so during the 15 years of follow-up: standardised incidence ratio 1.04 (0.74 to 1.43$)$ for all attempts and 0.97 (0.64 to 1.40 ) for first attempts. Twelve (38\%) of 32 patients who made their first suicide attempt before treatment made a new attempt or committed suicide thereafter. In contrast, $10(71 \%)$ of the 14 who made their first suicide attempt within six months after treatment stopped made a new attempt or committed suicide during follow-up (two sample test of proportions, $\mathrm{P}=0.034$ ). The number needed to harm was 2300 new six month treatments per year for one additional first suicide attempt to occur and 5000 per year for one additional repeat attempt.

Conclusions An increased risk of attempted suicide was apparent up to six months after the end of treatment with isotretinoin, which motivates a close monitoring of patients for suicidal behaviour for up to a year after treatment has ended. However, the risk of attempted suicide was already rising before treatment, so an additional risk due to the isotretinoin treatment cannot be established. As patients with a history of suicide attempts before treatment made new attempts to a lesser extent than did patients who started such behaviour in connection with treatment, patients with severe acne should not automatically have isotretinoin treatment withheld because of a history of attempted suicide.

\section{INTRODUCTION}

Isotretinoin (13-cis-retinoic acid) has been used since the 1980 s to treat severe recalcitrant nodular acne with good effect, but case reports and spontaneous reporting of adverse drug reactions have suggested an association between isotretinoin, depression, and suicidal behaviour. ${ }^{1-3}$ Observational studies have had conflicting results, however. ${ }^{4-6}$ Because a causal relation between isotretinoin and psychiatric reactions was difficult to exclude, labelling changes strengthening warnings about psychiatric adverse reactions have been added in product information for isotretinoin products within the EU despite contradictory evidence.

The association between acne, psychiatric morbidity, and suicide attempts has been described, ${ }^{7-11}$ and some authors have observed that isotretinoin actually leads to an improvement in anxiety and depression because of the clearing of disfiguring acne. ${ }^{12-16}$ Acne in itself is not considered to be a serious condition. If its treatment is suspected to entail severe side effects, the harm-benefit balance of that treatment must be thoroughly investigated. New data suggesting plausible biological mechanisms elucidating the association between retinoid regulation and mood through monoaminergic systems have been emerging. ${ }^{17}$

Our underlying hypothesis was that people with severe acne may be at a higher risk of attempted suicide regardless of exposure to isotretinoin. The aim of this study was to assess the risk of suicide attempts before, during, and after treatment with isotretinoin for severe acne.

\section{METHODS}

Identification of study population

The study population consisted of patients prescribed isotretinoin for severe acne between 1980 and 1990, 
who were aged 15 to 49 years at the first prescription. Isotretinoin is not registered in Sweden, and it can be dispensed only within a compassionate use programme after special application to the Medical Products Agency. We identified the study population in the register of named patients supplied the drug.

The individual application for a prescription holds information on prescribed dosage and total quantity; we used this to estimate the duration of treatment. We used the date of granting the prescription as a proxy for the first date of treatment. We considered patients to be exposed from the date of granting of the prescription and for the number of days for which the prescribed quantity at the prescribed dosage would suffice. For applications for which information on dosage was missing (197 patients, 3.4\%), we used the sex specific median daily dose. For 27 prescriptions, the total quantity was missing; for these we used the sex specific median quantity.

Starting in 1986-7, some dermatological clinics could be granted a "clinic approval" to prescribe isotretinoin without individual applications on condition that the requirements for individual applications were met. We identified patients prescribed isotretinoin with clinic approval in two steps. Each clinic approval was stored at a given single pharmacy. We asked these pharmacies to submit the lists of patients filling these prescriptions, together with the quantity of isotretinoin dispensed. However, these lists held only the names and dates of birth of the patients - the unique personal identification numbers were not registered. We then sent the lists obtained from the pharmacies to the clinics that had received the clinic approval, and they provided information on personal identity number and on actual starting and stopping dates of treatment as well as prescribed daily dose. The exact duration of treatment was registered for 1441 (25\%) of the 5756 patients in the cohort; for the remaining population, we estimated it from the prescribed amount and daily dose as stated in the individual applications. We did sensitivity analyses on the source of information (individual application versus clinic approval) about exposure.

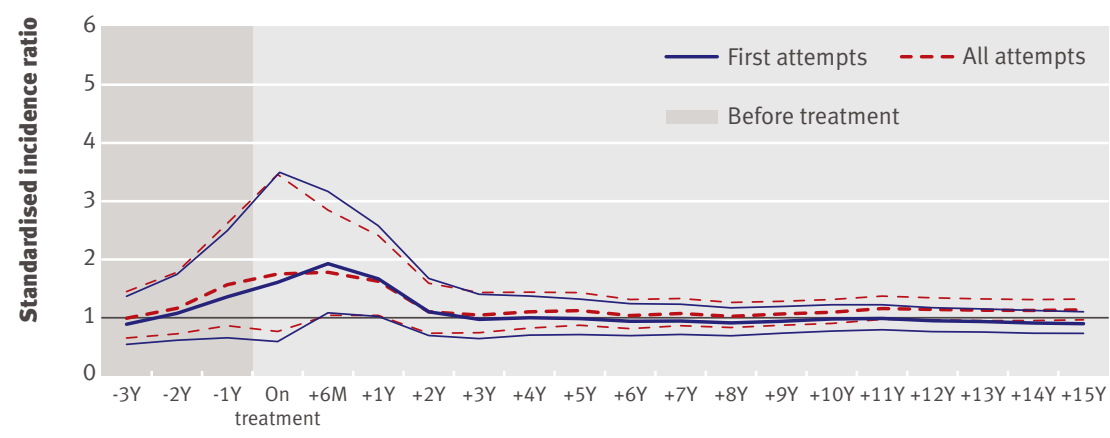

Cumulative No

$\begin{array}{lllllllllllllllllllll}\text { First } & 20 & 16 & 10 & 6 & 15 & 20 & 22 & 28 & 37 & 44 & 49 & 56 & 60 & 68 & 77 & 84 & 86 & 90 & 92 & 94\end{array}$ $\begin{array}{llllllllllllllllllllllll}\text { All } & 27 & 21 & 14 & 8 & 17 & 24 & 28 & 38 & 52 & 65 & 71 & 85 & 92 & 106 & 119 & 136 & 143 & 149 & 155 & 161\end{array}$

Fig 1 1 Standardised incidence ratios for accumulated first suicide attempts and all attempts from up to three years before (shaded area) to up to 15 years after treatment in all patients. Thin lines show $95 \%$ confidence intervals
Identification and classification of suicide attempts and deaths

We used the unique personal identification number to link patients in the study population to the national patient register of in-hospital care and to the cause of death register, both maintained by the National Board of Health and Welfare (www.socialstyrelsen.se). We extracted hospital admissions for suicide attempts, identified by codes for suicide attempt (ICD-8 (international classification of diseases, 8th revision) and ICD-9 codes E950-E958; ICD-10 codes X60-X64) and accidents with unclear intent (ICD-8 and ICD-9 codes E980-E988; ICD-10 codes Y10-Y34) entered as "external cause," as well as all deaths, with underlying causes, occurring between 1980 and 2001. We did sensitivity analyses excluding accidents with unclear intent.

We classified suicide attempts as occurring before, during, or after treatment. We counted events that occurred before treatment cumulatively for 12 month periods backwards in time up to three years before treatment. We restarted the cumulative counting of events at the start of treatment and counted events during the time of treatment, cumulatively for six months thereafter, and then cumulatively in consecutive 12 month periods up to 15 years after treatment.

\section{Time of observation and censoring}

The total time of observation encompassed 17197 person years of observation for up to three years before treatment, 2905 person years during treatment, and 87120 person years thereafter. To distinguish between rates of first time and repeated suicide attempts, we did two sets of analyses: for first attempts, we censored patients at the first hospital admission for attempted suicide, and they did not contribute person time of follow-up after that. Patients who made a first suicide attempt before treatment thus did not contribute any exposed treatment time in the analyses. In contrast, in the analysis of the total number of suicide attempts, any patient who attempted suicide continued to contribute person time after that event, and we counted subsequent hospital admissions. For both these analyses, we censored patients at the time of death (for any cause) or at the end of follow-up (31 December 2001).

We chose not to define a "time at risk" after the end of treatment with isotretinoin. To identify any long term effects of treatment, we distinguished only between time on treatment and time thereafter.

\section{External and internal comparisons of rates of suicide attempts}

We compared the rates of attempted suicide in the different time windows before, during, and after treatment with those of the general population, by using the national patient register of in-hospital care. We used sex, age (in five year bands), and calendar year specific rates in the general population to calculate the expected number of events in the study population, specifically for each time window analysed, before, during, and after treatment. In the study population, 
we calculated the person time of follow-up used for estimating the expected number of events specifically for sex, for age in five year bands, for calendar year, and for time window. We then calculated the standardised incidence ratio as the ratio between the observed number of cases and the expected number of cases.

We also used a cohort crossover analysis to make an internal comparison within the treated population, between rates of attempted suicide one year before treatment with the highest observed rates during or after treatment. ${ }^{18}$ By calculating the difference between these rates, we could estimate the absolute risk difference, assuming that the entire increase in risk was due to the treatment. We then used these rates to calculate the number needed to harm.

We thus made two overall comparisons. Firstly, we compared the study cohort with the general population, enabling an estimate of relative differences in the occurrence of suicide attempts between the study population and the background population. Secondly, an internal comparison within the study population gave an estimate of the absolute differences in risk before, during, and after treatment with isotretinoin for severe acne.

\section{Deaths by suicide}

In a separate analysis, we analysed the rates of completed suicides. The expected number of suicides came from mortality data for the entire general population, and we calculated mortality rates, standardised by sex, age (in five year bands), and calendar year. The causes of death, for both the study population and the general population, came from the national cause of death register. We used Stata for statistical analyses.

\section{RESULTS}

The cohort comprised 5756 people, of whom 3613 $(63 \%)$ were male. The mean age of male patients at first prescription was 22.3 (SD 6.6) years, and that of female patients was $27.1(8.0)$ years. The mean length of treatment was 6.0 (SD 4.0) months for male patients and 6.1 (3.9) months for female patients. The total time

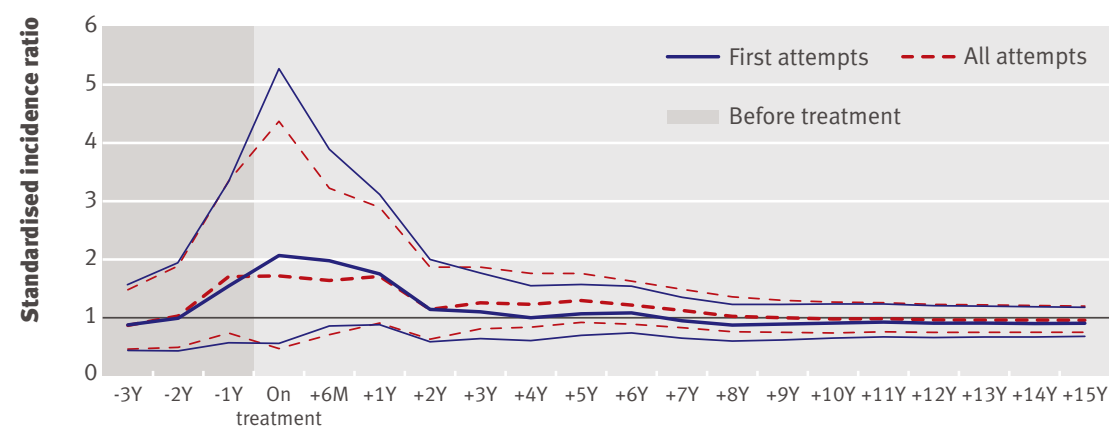

Cumulative No

$\begin{array}{lllllllllllllllllllll}\text { First } & 11 & 8 & 6 & 4 & 8 & 11 & 12 & 17 & 20 & 26 & 31 & 31 & 32 & 36 & 40 & 44 & 46 & 49 & 51 & 53\end{array}$ $\begin{array}{lllllllllllllllllllll}\text { All } & 13 & 10 & 8 & 4 & 8 & 13 & 15 & 24 & 31 & 40 & 45 & 48 & 50 & 54 & 58 & 63 & 66 & 69 & 72 & 74\end{array}$

Fig 2 | Standardised incidence ratios for accumulated first suicide attempts and all attempts from up to three years before (shaded area) to up to 15 years after treatment in male patients. Thin lines show $95 \%$ confidence intervals
Table 1|Patients' demographics and treatment characteristics. Values are numbers (percentages) unless stated otherwise

\begin{tabular}{|c|c|c|}
\hline Characteristics & $\begin{array}{c}\text { Males } \\
(n=3613)\end{array}$ & $\begin{array}{l}\text { Females } \\
(n=2143)\end{array}$ \\
\hline \multicolumn{3}{|l|}{ Age (years) at first prescription: } \\
\hline Mean (SD) & $22.3(6.6)$ & $27.1(8.0)$ \\
\hline Median (interquartile range) & $20(18-25)$ & $26(21-33)$ \\
\hline \multicolumn{3}{|l|}{ Prescribed daily doses (mg): } \\
\hline Mean (SD) & $44.5(15.7)$ & $39.2(13.1)$ \\
\hline Median (interquartile range) & $40(30-60)$ & $40(30-50)$ \\
\hline Missing & $129(3.6)$ & $68(3.2)$ \\
\hline \multicolumn{3}{|l|}{ Duration of treatment (months): } \\
\hline Mean (SD) & $6.0(4.0)$ & $6.1(3.9)$ \\
\hline Median (interquartile range) & $5.0(3.9-6.6)$ & $4.9(4.0-6.6)$ \\
\hline Total No of person years on treatment & 1819 & 1091 \\
\hline \multicolumn{3}{|l|}{ No of treatment courses: } \\
\hline 1 & 3320 (91.9) & 1956 (91.3) \\
\hline 2 & $272(7.5)$ & $175(8.2)$ \\
\hline 3 & $19(0.5)$ & $10(0.5)$ \\
\hline 4 & $2(0.1)$ & $2(0.1)$ \\
\hline
\end{tabular}

of treatment was 1819 person years for male patients and 1091 person years for female patients (table 1). More than $90 \%$ of patients were prescribed only one course of treatment.

Between 1980 and 2001, 128 (2.2\%) patients were discharged from in-hospital care at least once after a suicide attempt, of whom $70(1.9 \%)$ were male and 58 $(2.7 \%)$ were female. In total, 210 discharges were registered (1.6 per person). Of the suicide attempts, $85 \%$ were non-violent poisonings and overdoses (table 2).

Suicide attempts in isotretinoin cohort in relation to general population

Between three years and one year before the start of treatment, the standardised incidence ratios, comparing the study cohort with the general population, gradually rose from 0.89 (95\% confidence interval 0.54 to 1.37) three years before treatment to 1.36 (0.65 to 2.50) in the year before treatment for first attempts and from 0.99 (0.65 to 1.44$)$ three years before treatment to 1.57 ( 0.86 to 2.63$)$ in the year preceding treatment for all attempts. The risks were highest within six months after the start of treatment: 1.93 (1.08 to 3.18) for first attempts and 1.78 (1.04 to 2.85) for all attempts. The standardised incidence ratios then decreased, and the number of observed attempts was close to the expected number within three years after treatment: standardised incidence ratio $0.97(0.64$ to 1.40$)$ for first attempts and 1.04 (0.74 to 1.43) for all attempts (fig 1).

Eight years after treatment, the standardised incidence ratio for all attempts started to rise slightly above one, but this only occurred for female patients. Whereas the standardised incidence ratios for both first and repeat events were close to or below one for male patients (fig 2), those for repeat events in female patients (fig 3) were significantly raised 11 years after treatment $(1.36,1.06$ to 1.70$)$. We saw no such increase for first attempts in female patients. 
Table 2 |Details of suicide attempts and completed suicides by sex and by timing of events in relation to treatment

\begin{tabular}{|c|c|c|c|c|c|c|}
\hline & \multicolumn{2}{|c|}{ Ever before treatment } & \multicolumn{2}{|c|}{ During treatment plus 1 year } & \multicolumn{2}{|c|}{$\$ 1-15$ years after treatment } \\
\hline & Males & Females & Males & Females & Males & Females \\
\hline \multicolumn{7}{|l|}{ Suicide attempts } \\
\hline No of patients & 15 & 17 & 11 & 9 & 44 & 32 \\
\hline \multicolumn{7}{|l|}{ Age (years) at first event: } \\
\hline Mean & 23.2 & 26.0 & 25.6 & 27.4 & 32.3 & 33.7 \\
\hline Median (interquartile range) & $22(19-25)$ & $27(19-33)$ & $24(19-29)$ & $26(19-35)$ & $32(27-36)$ & $33(28-39)$ \\
\hline Range & $15-44$ & $15-38$ & $18-43$ & $17-42$ & $18-48$ & $19-49$ \\
\hline \multicolumn{7}{|l|}{ Method*: } \\
\hline Non-violent & 12 & 14 & 9 & 8 & 38 & 28 \\
\hline Violent & 3 & 3 & 3 & 1 & 5 & 4 \\
\hline \multicolumn{7}{|l|}{ Completed suicides } \\
\hline No of patients & NA & NA & 3 & 0 & 14 & 7 \\
\hline \multicolumn{7}{|l|}{ Age (years) at suicide: } \\
\hline Mean & NA & NA & 32.7 & NA & 33.5 & 35.6 \\
\hline Median (interquartile range) & NA & NA & $23 ; 37 ; 38 \dagger$ & NA & $32(29-41)$ & $37(31-40)$ \\
\hline Range & NA & NA & $23-38$ & NA & $21-46$ & $25-45$ \\
\hline \multicolumn{7}{|l|}{ Method*: } \\
\hline Non-violent & NA & NA & 1 & NA & 8 & 1 \\
\hline Violent & NA & NA & 2 & NA & 6 & 6 \\
\hline
\end{tabular}

$\mathrm{NA}=$ not applicable.

*Non-violent=poisoning, overdose; violent=cut/stab, jump, drowning, gas, firearm, suffocation.

†Actual ages of three patients who committed suicide.

Overall, we found no significant differences in prescribed daily doses or duration of treatment between people who attempted suicide and the cohort as a whole (table 3). However, the 14 female patients who attempted suicide after treatment had received two or more treatment courses significantly more often $(24 \%, 95 \%$ confidence interval $13 \%$ to $35 \%$ ) than had the female patients never admitted for attempted suicide $(9 \%, 8 \%$ to $10 \%)$ ( $\mathrm{P}<0.001$, two sample test of proportions).

Patients who made their first suicide attempt before treatment $(\mathrm{n}=32)$ did not seem to have their suicidal behaviour reinforced during or after treatment: 12 $(38 \%, 21 \%$ to $54 \%)$ of these patients made new attempts or committed suicide during the follow-up. In contrast, among the 14 who made a first suicide attempt during treatment or within six months after the end of

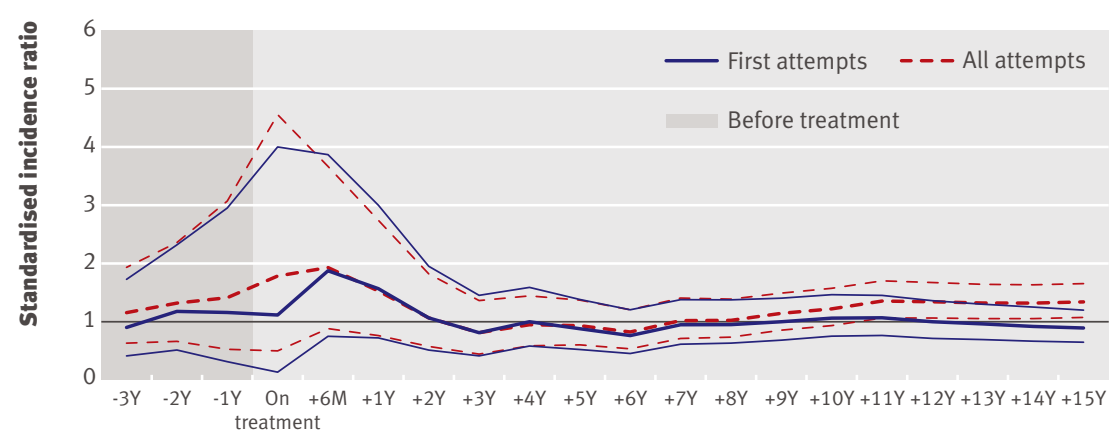

Cumulative No

$\begin{array}{lllllllllllllllllllll}\text { First } & 9 & 8 & 4 & 2 & 7 & 9 & 10 & 11 & 17 & 18 & 18 & 25 & 28 & 32 & 37 & 40 & 40 & 41 & 41 & 41\end{array}$ $\begin{array}{lllllllllllllllllllll}\text { All } & 14 & 11 & 6 & 4 & 9 & 11 & 13 & 14 & 21 & 25 & 26 & 37 & 42 & 52 & 61 & 73 & 77 & 80 & 83 & 87\end{array}$

Fig $3 \mid$ Standardised incidence ratios for accumulated first suicide attempts and all attempts from up to three years before (shaded area) to up to 15 years after treatment in female patients. Thin lines show $95 \%$ confidence intervals treatment, $10(71 \%, 48 \%$ to $95 \%)$ made a new attempt or committed suicide during follow-up $(\mathrm{P}=0.034$, two sample test of proportions).

Suicide attempts—comparisons within isotretinoin cohort As we saw when comparing the cohort with the general population, the rates of attempted suicide per person years of follow-up rose from three years before treatment and through to the actual treatment when we made internal comparisons within the treated cohort (fig 4). The highest rate difference was for first attempts: 0.86 (95\% confidence interval -0.78 to 2.50 ) cases per 1000 person years, comparing the rate during the year before treatment $(1.66 / 1000$ person years) with the rate within six months after treatment $(2.52 / 1000$ person years). For all attempts, the corresponding rate difference was $0.40(-1.46$ to 2.26$)$ per 1000 person years.

These rate differences yielded a number needed to harm of 2300 new six month treatments per year for one additional first suicide attempt to occur and 5000 per year for one additional repeat attempt, assuming that the entire additional risk could be attributed to the treatment. For both first and repeated suicide attempts, the rates and rate differences were higher for female patients than for male patients (fig 5 and fig 6 ).

\section{Death by suicide}

Seventeen male patients and seven female patients committed suicide by the end of $2001(0.4 \%)$. One man committed suicide during treatment and two within one year after treatment. The first woman who committed suicide did so six years after treatment.

Of the suicides, $42 \%$ were non-violent poisonings and overdoses (table 2) and $13(54 \%)$ of the patients 
Table $3 \mid$ Prescribed daily doses, duration of treatment, and number of treatment courses: total study population and patients admitted to hospital for suicide attempt, by sex. Values are numbers (percentages) unless stated otherwise

\begin{tabular}{|c|c|c|}
\hline & Males & Females \\
\hline \multicolumn{3}{|l|}{ Prescribed daily dose (mean (SD) mg) } \\
\hline All & $44.5(15.7)$ & $39.2(13.1)$ \\
\hline Suicide attempt, treatment +12 months & 35.0 & 40.0 \\
\hline Suicide attempt, treatment +15 years & 43.4 & 38.1 \\
\hline Suicide attempt, more than once & 39.4 & 38.4 \\
\hline \multicolumn{3}{|l|}{ Length of treatment (mean (SD) days) } \\
\hline All & $183.9(122.4)$ & $185.9(120.0)$ \\
\hline Suicide attempt, treatment + 12 months & 200.3 & 222.9 \\
\hline Suicide attempt, treatment +15 years & 201.5 & 204.6 \\
\hline Suicide attempt, more than once & 188.6 & 240.0 \\
\hline \multicolumn{3}{|l|}{ No of treatment courses } \\
\hline \multicolumn{3}{|l|}{ All: } \\
\hline 1 & $3320(91.9)$ & $1956(91.3)$ \\
\hline 2 & $272(7.5)$ & $175(8.2)$ \\
\hline 3 & $19(0.5)$ & $10(0.5)$ \\
\hline 4 & $2(0.1)$ & $2(0.1)$ \\
\hline \multicolumn{3}{|l|}{ Suicide attempt, treatment + 15 years: } \\
\hline 1 & $63(90)$ & $44(76)$ \\
\hline 2 & $5(7)$ & $13(22)$ \\
\hline 3 & $2(3)$ & $1(2)$ \\
\hline 4 & 0 & 0 \\
\hline
\end{tabular}

had made suicide attempts before the completed suicide. The standardised mortality ratio for male patients who committed suicide within one year after treatment $(\mathrm{n}=3)$ was 1.9 (0.4 to 5.4). Within two years after treatment, the standardised mortality ratios for suicide among male patients went down to around one- that is, the observed number was close to the expectedand remained there throughout the observation period (data not shown). The highest standardised mortality ratio for female patients occurred within 11 years after treatment: six suicides yielded a ratio of 1.8 (0.7 to 3.9).

\section{Sensitivity analyses}

We calculated separate incidences depending on the source of information about exposure. We made one estimation for patients with durations of treatment estimated from dosage and quantity stated in the applications for prescriptions and one for patients with known durations of treatments obtained from the clinics. The patterns of incidences before, during, and after treatment were identical, and the point estimates were very similar. We found no differences in the patterns of incidences when we excluded accidents with unclear intent.

\section{DISCUSSION}

This retrospective cohort study of 5756 patients treated with isotretinoin for severe acne encompasses more than 17000 person years of observation before treatment, 2900 person years on treatment, and 87000 person years of follow-up thereafter. During the observation period, 128 patients were admitted to hospital for attempted suicide on 210 occasions; 24 patients committed suicide. The data support our hypothesis that severe acne, regardless of exposure to isotretinoin, carries an increased risk of attempted suicide. The point estimates of the standardised incidence ratios rose gradually from three years before treatment through to the year immediately before treatment. However, none of these point estimates was statistically significant. The risk was significantly raised only six months after the end of treatment. Two to three years after treatment, the observed number of suicide attempts was close to the expected number, given the background rate in the population.

One condition for prescription of isotretinoin being allowed was that the patient had been treated with tetracyclines for at least six months without improvement. The increasing risk of attempted suicide during the year before treatment with isotretinoin coincides with the presumed treatment with tetracyclines. We are not aware, however, of any hypotheses or signals associating tetracycline with psychiatric side effects, including suicide attempts.

Considering the increasing risk of attempted suicide during the years before treatment, we cannot state whether the continued rise during and immediately after treatment was due to the natural course of severe acne or to negative effects of the treatment. As the standardised incidence ratio went down to one within two to three years after treatment, a successful treatment could - at the population level—have actually attenuated the suicidal behaviour due to severe acne. Some of the patients, possibly vulnerable to isotretinoin, who made their first suicide attempt in close relation to treatment, may have done so as a consequence of exposure to the drug. However, patients without a positive effect of treatment might have despaired at the prospect of continuing to live with disfiguring acne. Furthermore, we cannot exclude the possibility that patients whose acne improved after treatment were distraught if no improvement in their social life followed the physical improvement. We must stress that we cannot exclude the possibility that the raised risk of suicide attempts during treatment and six months after treatment is

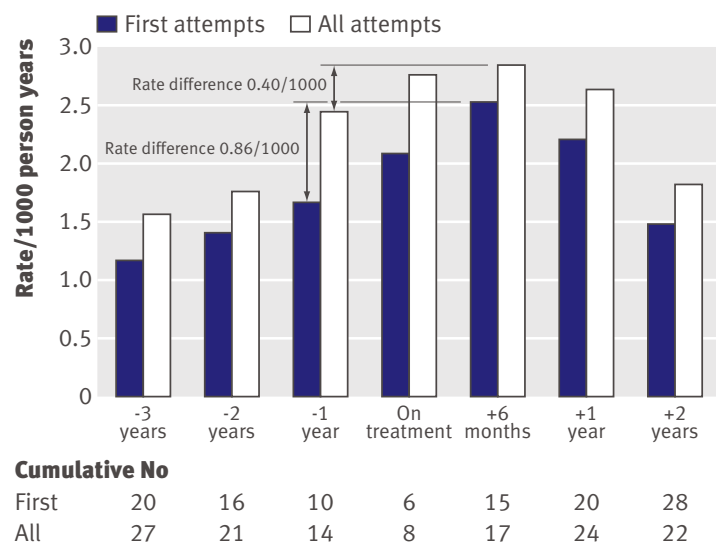

Fig 4 | Rates per 1000 person years of follow-up for first suicide attempts and all attempts from up to three years before and up to two years after treatment for all patients 


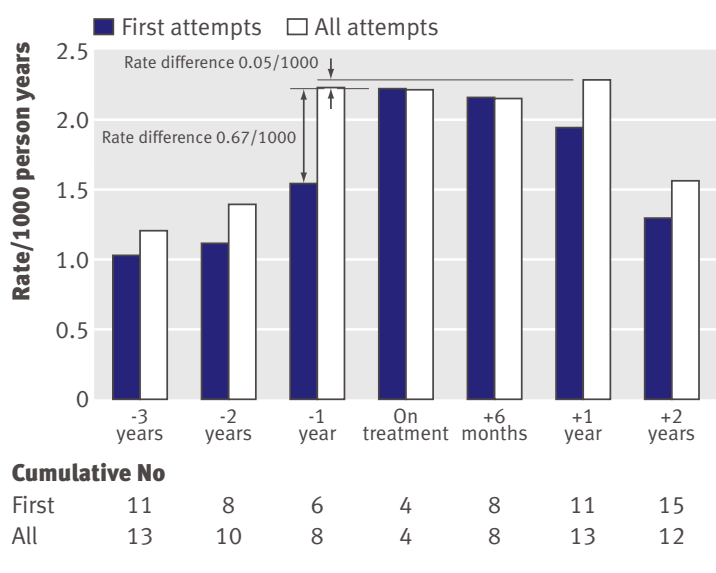

Fig 5 | Rates per 1000 person years of follow-up for first suicide attempts and all attempts from up to three years before and up to two years after treatment for male patients

due to the exposure to isotretinoin. However, a more probable interpretation is that the underlying severe acne may best explain the raised risk.

Although we do not know if some patients in the study population were also treated with isotretinoin after 1990, we could detect no long term effects of the treatment on the risk of patients starting suicidal behaviour. However, the standardised incidence ratio for repeated attempts increased among female patients eight years after treatment. Whether this is due to new episodes of treatment or to the presumed higher psychiatric vulnerability of women to severe acne is unknown. ${ }^{19}$

Patients who made suicide attempts before treatment did not seem to have this behaviour reinforced by treatment with isotretinoin. Patients with acne who made their first suicide attempt in connection with isotretinoin treatment made new attempts approximately twice as often as did those who made their first suicide attempt before the exposure to isotretinoin. One possible explanation for this difference may be that patients with an assumed acne induced suicidality had an improvement in their severe acne after a successful treatment. In those patients who made a first suicide attempt during or soon after the treatment, this behaviour could be a side effect of the treatment itself or a consequence of treatment failure or of lack of perception of improving quality of life despite successful treatment. A finding in line with this is that women who made suicide attempts received two or three treatments more often than did women who did not make suicide attempts. This indicates treatment failure as a possible explanation for suicidal behaviour. This may indicate that a history of suicide attempts need not be a decisive factor when considering treatment with isotretinoin.

Attempted suicide is, however, an uncommon event. In the internal comparison of the cohort, the number needed to harm was 2300 per year for one additional first suicide attempt and 5000 per year for one additional repeat attempt to occur, assuming that the entire increase in risk was due to treatment. A physician would thus need to start treatments in more than 2000 new patients during one year to see one additional suicide attempt due to treatment. Nevertheless, the most important proactive measure to be taken would be to closely monitor all patients' psychiatric status, not only during treatment but also for at least a year after treatment with isotretinoin.

\section{Comparisons with previous studies and findings}

Many case reports and case series have reported serious adverse psychiatric events associated with isotretinoin treatment. ${ }^{20}$ In epidemiological studies, the results have been conflicting. No association was found between isotretinoin treatment and depression, psychotic symptoms, suicide, or suicide attempts in a cohort of more than 7000 isotretinoin users. ${ }^{4}$ On the other hand, a significant relative risk of 2.68 (95\% confidence interval 1.10 to 6.48 ) was found in 126 patients with incident depression and acne in a case crossover study. ${ }^{6}$ Furthermore, 1419 army conscripts treated with isotretinoin for acne were found to use mental health services more than did a control group with psoriasis. $^{5}$

In several prospective studies, treatment with isotretinoin has been shown to improve patients with acne emotionally and psychologically. ${ }^{12-17}$ However, these studies were probably too small to detect negative events with incidences in the order of 1-2 per 1000 person years. Many studies have shown that acne in itself has a negative psychological impact. ${ }^{7-11}$ For example, in line with our findings, severe acne has been shown to be associated with suicidal behaviour. ${ }^{10}$

The standardised incidence ratios and rates of attempted suicide were higher in female than in male patients; a stronger emotional impact of acne in girls has also been observed previously. ${ }^{19} \mathrm{We}$ also observed that women who made suicide attempts after treatment had more often received two or more treatment courses with isotretinoin than had women who did not make suicide attempts. This indicates that they

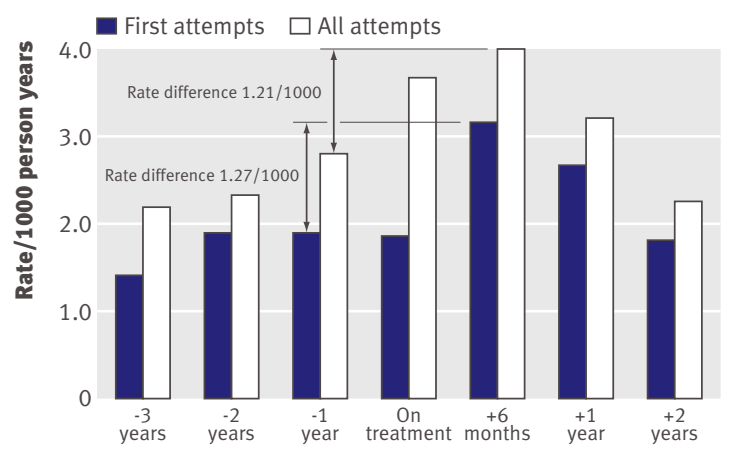
Cumulative No

$\begin{array}{lccccccc}\text { First } & 9 & 8 & 4 & 2 & 7 & 9 & 10 \\ \text { All } & 14 & 11 & 6 & 4 & 9 & 11 & 13\end{array}$

Fig 6 | Rates per 1000 person years of follow-up for first suicide attempts and all attempts from up to three years before and up to two years after treatment for female patients 
may have had more severe, treatment resistant acne or that the emotional impact of severe acne may be more pronounced in female patients.

Several studies have shown that the molecular components needed for retinoic acid signalling are expressed in the adult brain, suggesting that retinoids could play a role in affective disorders. ${ }^{21-29}$ In young adult mice, chronic administration of isotretinoin induced depression related behaviours. ${ }^{22}$ These changes were not seen in adult rats. ${ }^{27}$ In 13 humans, isotretinoin decreased orbitofrontal cortex metabolism, but this decrease was not accompanied by an increase in the severity of depression. ${ }^{28}$

\section{Limitations and strengths of study}

Our study has some limitations. Despite the large number of person years of exposure and follow-up, the statistical precision was low. The standardised incidence ratios were clearly rising before treatment, but we found no statistical significance until six months after treatment. In the internal cohort crossover analysisthat is, analysing outcomes before and after treatment in the same population - the differences in incidence did not reach statistical significance, making the estimated number needed to harm uncertain. We estimated the exposure to isotretinoin from prescriptions, not from actual use. However, during the 1980 s, isotretinoin was a newly available drug for the treatment of severe acne. One requirement for prescribing the drug was that the patient had been treated with antibiotics for at least six months without improvement. This would have made this population motivated for treatment, and thus likely to have complied with it.

After 1986-7, dermatology clinics could prescribe isotretinoin without an individual application for each patient. We could not collect the identities of all patients treated at such clinics. These unidentified patients would, however, be a source of bias only if the frequency/pattern of suicide attempts can be assumed to be distinctly different in that "lost" population. We cannot conceive of a mechanism to justify such an assumption.

We had no information on the effect of treatment. A bias would exist if the patients who made suicide attempts had a poorer effect of treatment than did those who did not make such attempts. In that case, the suicidal behaviour should be attributed to the treatment resistant acne and not to the treatment. However, such bias would only strengthen the assumed association between severe acne and suicidal behaviour.

Loss to follow-up is a minor source of error, as only people who emigrated from Sweden were truly lost to follow-up. A bias would result if patients treated with isotretinoin emigrated and made a suicide attempt thereafter. This would most probably lead to our observed standardised incidence ratio being underestimated.

No potential confounding factors other than age, sex, and calendar year were available for analysis. The cohort crossover analysis none the less attenuated the effect from unmeasured confounding factors, as we compared the exposed population with itself before treatment. On the other hand, we could not account or control for effects of risk factors changing within a person over time. For example, evidence exists of an association between unemployment and attempted suicide, as well as between divorce and suicide/suicidal behaviour. ${ }^{30-32}$ As regards the external comparison with the general population, our risk estimates would be overestimated if the rates of unemployment or separation were higher in the exposed population. In the internal comparison of rates before, during, and after treatment, the population aged over time. Separations and divorces may well have been increasing with age in the cohort. The rising rates of attempted suicide before and immediately after treatment would then have been overestimated as a result of this bias. However, in the period after treatment, the decline in these rates would have been underestimated if the rates of separations continued to rise with time. We consider that any unmeasured confounder changing intraindividually over time would have to correlate with the increasing severity of acne, as well as with treatment and its end, to explain the observed results. Such strong correlations are difficult to imagine.

In this study, we had data only on serious suicide attempts leading to admission to hospital. Although including such serious attempts can be considered a strength, the fact that we could not include patients who made less severe suicide attempts and who were treated as outpatients, or who were not in contact at all with health services, is also a possible limitation. This would, however, mean that we have underestimated the incidence of attempted suicide in our study, possibly to a greater extent before treatment than during and after treatment. Undocumented suicide attempts may have occurred more often before treatment than during and after treatment, as treatment with isotretinoin required an established contact with specialised healthcare services, and this may have made such patients more willing to seek medical help for suicidal ideation or attempted suicide.

We used the same register to identify the suicide attempts within the cohort that we used to calculate the expected number of events. No bias in identification of cases depending on exposure is thus probable. Furthermore, this cohort should be close to representing $100 \%$ of the treated population in Sweden up to 1986, and a high proportion thereafter, and it was followed for up to 15 years after treatment. The two comparisons - with the general population and internally within the cohort-gave the same results regarding the pattern of incidence of suicide attempts. The analyses of standardised incidence ratios and rates before treatment both confirm the existence, and give an estimation of the extent, of the usually so elusive "confounding by indication."

\section{Conclusions and implications}

Severe acne is not a trivial condition; in the absence of treatment with isotretinoin, it is associated with an increased risk of attempted suicide. At the level of the 


\section{WHAT IS ALREADY KNOWN ON THIS TOPIC}

Case reports and spontaneous reports of adverse drug reactions have suggested an association between isotretinoin and suicidal behaviour, but observational studies have had conflicting results

Isotretinoin has been shown to have behavioural effects in mice and effects on cerebral metabolism in humans

\section{WHAT THIS STUDY ADDS}

Severe acne is a risk factor for attempted suicide; an additional risk may be present during and up to one year after treatment with isotretinoin

Suicidal behaviour triggered during isotretinoin treatment more often leads to repeated attempts or suicide than in patients with a history of suicide attempts before treatment

A history of attempted suicide may not need to be a contraindication when considering treatment with isotretinoin
7 Gupta MA, Gupta AK. Psychiatric and psychological co-morbidity in patients with dermatologic disorders: epidemiology and management. Am J Clin Dermatol 2003;4:833-42.

8 Yazici K, Baz K, Yazici AE, Kokturk A, Tot S, Demirseren D, et al. Disease-specific quality of life is associated with anxiety and depression in patients with acne. J Eur Acad Dermatol Venereol 2004;18:435-9.

9 Magin PJ, Pond CD, Smith WT, Watson AB, Goode SM. A crosssectional study of psychological morbidity in patients with acne, psoriasis and atopic dermatitis in specialist dermatology and general practices. J Eur Acad Dermatol Venereol 2008;22:1435-44.

10 Purvis D, Robinson E, Merry S, Watson P. Acne, anxiety, depression and suicide in teenagers: a cross-sectional survey of New Zealand secondary school students. J Paediatr Child Health 2006;42:793-6.

11 Smithard A, Glazebrook C, Williams HC. Acne prevalence, knowledge about acne and psychological morbidity in mid-adolescence: a community-based study. Br J Dermatol 2001;145:274-9.

12 Chia CY, Lane W, Chibnall J, Allen A, Siegfried E. Isotretinoin therapy and mood changes in adolescents with moderate to severe acne: $a$ cohort study. Arch Dermatol 2005;141:557-60.

13 Kellett SC, Gawkrodger DJ. A prospective study of the responsiveness of depression and suicidal ideation in acne patients to different phases of isotretinoin therapy. Eur J Dermatol 2005;15:484-8.

14 Kaymak Y, Kalay M, Ilter N, Taner E. Incidence of depression related to isotretinoin treatment in 100 acne vulgaris patients. Psychol Rep 2006;99:897-906.

15 Kaymak Y, Taner E, Taner Y. Comparison of depression, anxiety and life quality in acne vulgaris patients who were treated with either isotretinoin or topical agents. Int J Dermatol 2009;48:41-6.

16 Hahm BJ, Min SU, Yoon MY, Shin YW, Kim JS, Jung JY, et al. Changes of psychiatric parameters and their relationships by oral isotretinoin in acne patients. J Dermatol 2009;36:255-61

17 O'Reilly K, Bailey SJ, Lane MA. Retinoid-mediated regulation of mood: possible cellular mechanisms. Exp Biol Med (Maywood) 2008;233:251-8.

18 Marcil I, Stern RS. Squamous-cell cancer of the skin in patients given PUVA and ciclosporin: nested cohort crossover study. Lancet 2001;358:1042-5.

19 Aktan S, Ozmen E, Sanli B. Anxiety, depression, and nature of acne vulgaris in adolescents. Int J Dermatol 2000;39:354-7.

20 Kontaxakis VP, Skourides D, Ferentinos P, Havaki-Kontaxaki BJ, Papadimitriou GN. Isotretinoin and psychopathology: a review. Ann Gen Psychiatry 2009;8:2.

21 O'Reilly KC, Shumake J, Bailey SJ, Gonzalez-Lima F, Lane MA. Chroni 13-cis-retinoic acid administration disrupts network interactions between the raphe nuclei and the hippocampal system in young adult mice. Eur J Pharmacol 2009;605:68-77.

22 O'Reilly KC, Shumake J, Gonzalez-Lima F, Lane MA, Bailey SJ. Chroni administration of 13-cis-retinoic acid increases depression-related behavior in mice. Neuropsychopharmacology 2006;31:1919-27.

23 O’Reilly KC, Trent S, Bailey SJ, Lane MA. 13-cis-retinoic acid alters intracellular serotonin, increases 5-HT1A receptor, and serotonin reuptake transporter levels in vitro. Exp Biol Med (Maywood) 2007;232:1195-203.

24 Crandall J, Sakai Y, Zhang J, Koul O, Mineur Y, Crusio WE, et al. 13-cis retinoic acid suppresses hippocampal cell division and hippocampal-dependent learning in mice. Proc Natl Acad Sci U S A 2004;101:5111-6.

25 Ishikawa J, Sutoh C, Ishikawa A, Kagechika H, Hirano H, Nakamura S. 13-cis-retinoic acid alters the cellular morphology of slice-cultured serotonergic neurons in the rat. Eur J Neurosci 2008;27:2363-72.

26 Liu Y, Kagechika H, Ishikawa J, Hirano H, Matsukuma S, Tanaka K, et al. Effects of retinoic acids on the dendritic morphology of cultured hippocampal neurons. J Neurochem 2008;106:1104-16.

27 Ferguson SA, Cisneros FJ, Hanig JP, Berry KJ. Oral treatment with Accutane does not increase measures of anhedonia or depression in rats. Neurotoxicol Teratol 2007;29:642-51.

28 Bremner JD, Fani N, Ashraf A, Votaw JR, Brummer ME, Cummins T, et al. Functional brain imaging alterations in acne patients treated with isotretinoin. Am J Psychiatry 2005;162:983-91.

29 Bremner JD, McCaffery P. The neurobiology of retinoic acid in affective disorders. Prog Neuropsychopharmacol Biol Psychiatry 2008;32:315-31.

30 Stuckler D, Basu S, Suhrcke M, Coutts A, McKee M. The public health effect of economic crises and alternative policy responses in Europe: an empirical analysis. Lancet 2010;374: 315-23.

31 Wyder M, Ward P, De Leo D. Separation as a suicide risk factor. J Affect Disord 2009;116:208-13.

32 Kolves K, Ide N, De Leo D. Suicidal ideation and behaviour in the aftermath of marital separation: gender differences. J Affect Disord 2010;120: 48-53.

Accepted: 06 September 2010 Increased use of mental health senvices related to isotretinoin treatment: a 5-year analysis. Eur Neuropsychopharmacol 2006;16:413-6.

6 Azoulay L, Blais L, Koren G, LeLorier J, Berard A. Isotretinoin and the risk of depression in patients with acne vulgaris: a case-crossover study. J Clin Psychiatry 2008;69:526-32. 